\title{
Tumor-penetration and antitumor efficacy of cetuximab are enhanced by co-administered iRGD in a murine model of human NSCLC
}

\author{
YANG ZHANG $^{1,2}$, JIE YANG ${ }^{1}$, MANHUA DING $^{3}$, LIANTAO LI $^{1}$, \\ ZHENG LU $^{1}$, QING ZHANG ${ }^{1}$ and JUNNIAN ZHENG ${ }^{1,4}$ \\ ${ }^{1}$ Cancer Institute, Xuzhou Medical College, Xuzhou, Jiangsu 221002; ${ }^{2}$ Department of Oncology, \\ Xuzhou Central Hospital, Xuzhou, Jiangsu 221009; ${ }^{3}$ Department of Oncology, Xuzhou Tumor Hospital, \\ Xuzhou, Jiangsu 221005; ${ }^{4}$ Jiangsu Center for the Collaboration and Innovation of Cancer Biotherapy, \\ Cancer Institute, Xuzhou Medical College, Xuzhou, Jiangsu 221002, P.R. China
}

Received April 10, 2015; Accepted June 16, 2016

DOI: $10.3892 / 01.2016 .5081$

\begin{abstract}
Lung cancer is the leading cause of cancer-associated mortality, worldwide. For this reason, novel therapies are required for the treatment of this devastating disease. Cetuximab is a monoclonal antibody against epidermal grow th factor receptor (EGFR), which is overexpressed in a variety of solid tumors, including non-small cell lung cancer (NSCLC). The therapeutic efficacy of cetuximab for NSCLC is limited to use as a monotherapy or in combination with chemotherapy. The objective of the present study was to develop a novel strategy to enhance the therapeutic efficacy of cetuximab for NSCLC by a co-administration with the tumor-penetrating internalizing RGD peptide (iRGD). Human NSCLC subcutaneous xenograft models established with the A549 cell line in nude mice were treated with $30 \mathrm{mg} / \mathrm{kg}$ cetuximab, $4 \mathrm{mg} / \mathrm{kg}$ iRGD, cetuximab plus iRGD or phosphate-buffered saline. The tumor-penetration, in vivo therapeutic efficacy and involved mechanism were evaluated. The present study showed that the A549 xenograft model is sensitive to the co-administration of cetuximab and iRGD. Treatment with cetuximab plus iRGD
\end{abstract}

Correspondence to: Dr Qing Zhang or Professor Junnian Zheng, Cancer Institute, Xuzhou Medical College, West Campus of Xuzhou Medical College, 84 West Huaihai Road, Xuzhou, Jiangsu 221002, P.R. China

E-mail: zhangqing@xzmc.edu.cn

E-mail: jnzheng@xzmc.edu.cn

Abbreviations: NSCLC, non-small cell lung cancer; iRGD, internalizing RGD peptide; EGFR, epidermal growth factor receptor; p-EGFR, phosphorylated EGFR; CendR, cryptic C-end Rule; NRP-1, neuropilin-1; TUNEL, TdT-mediated dUTP nick end labeling kit

Key words: cetuximab, combination therapy, internalizing RGD peptide, tumor-penetrating peptide, non-small cell lung cancer resulted in a significant increase in the tumor-penetration of cetuximab and tumor reduction compared with cetuximab monotherapy. In conclusion, iRGD enhances the effects of co-administered cetuximab in an NSCLC model. The combined application of cetuximab and iRGD may be a novel strategy to enhance the clinical therapeutic efficacy of cetuximab for the treatment of NSCLC.

\section{Introduction}

Lung cancer is one of the most common malignant tumors to occur, worldwide. The two major subtypes are small cell lung cancer (SCLC) and non-small cell lung cancer (NSCLC) (1). NSCLC accounts for $\sim 85 \%$ of all lung cancer cases (2). For early-stage or locally advanced lung cancer, surgery is the most effective treatment and combined chemotherapy is the standard adjuvant approach. However, $40 \%$ of patients with NSCLC present with unresectable, metastatic stage IV tumors (3). The prognosis is poor, and the overall 5-year survival rate remains $<15 \%$ (2). Since the effectiveness of current standard treatment for advanced NSCLC (i.e. chemotherapy) has reached a ceiling (4), there is a continuous requirement for novel, more effective treatments to further improve the outcome of patients with the disease.

Epidermal growth factor receptor (EGFR) is a transmembrane glycoprotein that is overexpressed in numerous solid tumors, including NSCLC (5). The binding of a ligand, for example transforming growth factor- $\alpha$ or epidermal growth factor, to EGFR triggers tyrosine kinase phosphorylation, which in turn activates cellular pathways, including the mitogen-activated protein kinase, phosphatidylinositol $3^{\prime}$ kinase and protein kinase B pathways. The ultimate result is cell growth and tumor progression. Therefore, EGFR is an important therapeutic target in NSCLC (6).

Cetuximab (Erbitux ${ }^{\circledR}$ ) is a chimeric human-murine monoclonal immunoglobulin ( $\mathrm{Ig}) \mathrm{G} 1$ antibody. It blocks ligand binding to EGFR, leading to a decrease in receptor dimerisation, autophosphorylation and activation of signaling pathways (7). Cetuximab may also act by means 
of antibody-dependent cellular cytotoxicity and complement-dependent cytotoxicity (2). In 2004, the results of a single-agent phase II study of cetuximab in recurrent NSCLC were reported, with 29 EGFR-positive patients showing a partial response rate of $7 \%$ and a stable disease rate of $17 \%$ (8). Two randomized phase III trials that evaluated cetuximab in NSCLC patients showed a small benefit in terms of overall survival for the experimental treatment, which was considered insufficient by the European Medicines Agency for marketing approval (1). One of the possible reasons for limited therapeutic efficacy of cetuximab in NSCLC may be that the increased interstitial fluid pressure inside the tumor prevents the antibody from being delivered into the extravascular tumor tissue (9-12).

The tumor-targeted delivery of anticancer agents is considered as a novel strategy to traditional chemotherapy. Tumor-targeted delivery has been proved to selectively increase the drug concentration at the tumor site and significantly improve the therapeutic efficacy (13). Previously, a tumor-penetrating internalizing RGD peptide (iRGD; amino acid sequence, CRGDK/RGPD/EC), was identified and reported to increase vascular and tissue penetration in a tumor-specific and neuropilin-1 (NRP-1)-dependent manner (14). The RGD tripeptide of iRGD can bind the $\alpha v \beta 3$ and $\alpha v \beta 5$ integrins, the expression of which is largely restricted to tumors, including tumor vasculature and cells $(14,15)$. When iRGD binds to integrins, the peptide bond between the $\mathrm{K}$ and $\mathrm{G}$ amino acids is proteolytically cleaved by the cell surface-associated proteases to expose the cryptic C-end rule (CendR) motif (amino acid sequence, CRGDK/R), which then binds to NRP-1 (14-17). The activation of NRP-1 increases the permeability of blood vessels and tumor tissues, which allows drugs to penetrate into the internal tissue of the tumor much more easily $(14,15,18)$. Thus, when anticancer agents are modified with iRGD, the tissue-penetrating and tumor-targeting properties can be improved. Additionally, when iRGD was previously co-administered with drugs or a systemic injection drug delivery system, antitumor activities were also improved (18).

The characteristics of iRGD in solid cancers appear to warrant further investigation; particularly, for patients with diseases that have a dismal prognosis, such as NSCLC. However, there have been no comprehensive in vivo studies using NSCLC models, and the effects of iRGD used in combination with cetuximab have not yet been tested. Therefore, the present study examined the effectiveness of iRGD for boosting cetuximab accumulation in NSCLC xenograft models established with the A549 cell line. The anticancer booster effect of combination therapy with cetuximab plus iRGD was evaluated with in vivo experiments.

\section{Materials and methods}

Cell culture. The human NSCLC-derived A549 cell line was purchased from the Shanghai Institute of Biochemistry and Cell Biology, Chinese Academy of Sciences (Shanghai, China), and cultured in F-12K medium with $10 \%$ fetal bovine serum and $1 \%$ penicillin/streptomycin (all Gibco; Thermo Fisher Scientific, Inc., Waltham, MA, USA). Cells were cultured in a humidified atmosphere containing $5 \% \mathrm{CO}_{2}$ at $37^{\circ} \mathrm{C}$.
Flow cytometry. A total of $1 \times 10^{6}$ A549 cells were incubated with fluorescent-labeled antibodies diluted in $100 \mu \mathrm{l}$ phosphate-buffered saline (PBS) for $30 \mathrm{~min}$ at room temperature. The cells were then washed, suspended and evaluated with a FACS machine (FACSCanto II; BD Biosciences, Franklin Lakes, NJ, USA). A matched isotype control antibody was used in all analyses. Finally, all the data were analyzed by FlowJo 7.6.1 software (FlowJo, LLC, Ashland, OR, USA). Integrin $\alpha v \beta 3$ was detected using a fluorescein isothiocyanate (FITC)-conjugated mouse anti-human integrin $\alpha v \beta 3$ monoclonal antibody (1:100; cat. no. MAB1976; EMD Millipore, Billerica, MA, USA), and integrin $\alpha v \beta 5$ was detected using a FITC-conjugated mouse anti-human integrin $\alpha v \beta 5$ monoclonal antibody (1:100; cat. no. MAB1961F; EMD Millipore).

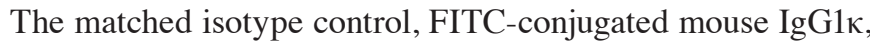
was purchased from eBioscience, Inc. (San Diego, CA, USA; 1:100; cat. no. 11-4714-41). NRP-1 was detected using a phycoerythrin (PE)-conjugated mouse anti-human NRP-1 monoclonal antibody and an isotype control (both 1:100; cat. nos. 130-098-876 and 130-098-845, respectively; Miltenyi Biotec GmbH, Bergisch Gladbach, Germany). EGFR was detected using a PE-conjugated mouse anti-human EGFR monoclonal antibody and an isotype control (both 1:100; cat. nos. 555997 and 555743, respectively; BD Biosciences).

Mice and in vivo experiments. In vivo experiments involved 69 six-week-old female BALB/C nude mice (Vital River Laboratory Animal Technology Co., Ltd, Beijing, China), which were housed in the specific pathogen-free animal facility of Experimental Animal Center, Xuzhou Medical College (Xuzhou, China). Animals were housed with a 12-h light/dark cycle, at $22 \pm 1^{\circ} \mathrm{C}$, and in a $55 \pm 5 \%$ humidity-controlled room. All mice were allowed free access to clean water and food. All cages housed up to 6 animals and contained wood shavings and an independent air supply system. All animal experimental protocols were approved and reviewed by the Institutional Animal Care and Use Committee of the Jiangsu Provincial Academy of Chinese Medicine (approval no., SCXK 2012-0005). During in vivo experiments, animals in all experimental groups were examined daily for physical activity. At the end of the experiment, mice were sacrificed by cervical dislocation.

Tumor model. The human NSCLC model was established as previously described (19). Briefly, $1 \times 10^{7}$ A549 cells were injected into the left forelimb armpits of $6 \mathrm{BALB} / \mathrm{c}$ nude mice. When the tumors grew to $\sim 150 \mathrm{~mm}^{3}$, the mice were sacrificed by cervical dislocation, and the tumors were harvested and segmented into tissue blocks (4-6 $\mathrm{mm}^{3}$ in size). Subsequently, the left forelimb armpits of three BALB/c nude mice were inoculated subcutaneously with the tissue blocks using a trocar. The mice were sacrificed when the tumors grew to the desired size.

Immunofluorescence staining. Three mice were used to establish human NSCLC models. When the tumors reached $\sim 200 \mathrm{~mm}^{3}$, the mice were sacrificed and the tumors were dissected and sectioned on a cryostat. For $\alpha v \beta 3, \alpha v \beta 5$ and NRP-1 detection, the sections $(n=3)$ were incubated overnight at $4^{\circ} \mathrm{C}$ with the same anti-human $\alpha v \beta 3, \alpha v \beta 5$ and NRP-1, or isotype control antibodies, as those used in the 
flow cytometry analysis. After washing, all sections were observed and photographed with a fluorescence microscope (DS-Ri1; Nikon Corporation, Toyko, Japan).

Tumor permeability assay. When tumors reached $200 \mathrm{~mm}^{3}$, the mice were divided into 4 groups ( $n=9 /$ group). A total of $30 \mathrm{mg} / \mathrm{kg}$ cetuximab (Merck Millipore, Darmstadt, Germany), $4 \mathrm{mg} / \mathrm{kg}$ iRGD (Shanghai Apeptide Co., Ltd., Shanghai, China), $30 \mathrm{mg} / \mathrm{kg}$ cetuximab+4 $\mathrm{mg} / \mathrm{kg}$ iRGD or PBS was injected into each of the 4 groups of tumor-bearing mice via the tail vein. After 1, 3 and 9 h, 3 mice per group were anesthetized by intraperitoneal injection with $3.5 \%$ chloral hydrate (0.2 ml/mouse; Shanghai Shifeng Biological Technology, Co., Ltd., Shanghai, China). After 2 min, the mice were perfused through the heart with PBS containing $1 \%$ bovine serum albumin (Sigma-Aldrich, St. Louis, MO, USA). The tumors were then collected and sectioned on a cryostat. The tumor vasculature marker cluster of differentiation (CD)31 was stained with a rabbit anti-mouse CD31 polyclonal antibody (cat. no. ab28364; Abcam, Cambridge, MA, USA) and a DyLight 549-conjugated goat anti-rabbit IgG $(\mathrm{H}+\mathrm{L})$ secondary antibody (cat. no. E032320; EarthOx Life Sciences, Millbrae, CA, USA) was used for visualization. Cetuximab was stained with the DyLight 488-conjugated goat anti-human IgG $(\mathrm{H}+\mathrm{L})$ antibody (cat. no. 109-485-003; Jackson ImmunoResearch Inc., West Grove, PA, USA). The nuclei were stained with 4',6-diamidino-2-phenylindole (Roche Diagnostics $\mathrm{GmbH}$, Mannheim, Germany). Imaging was performed using a confocal microscope (OLS4100; Olympus Corporation, Tokyo, Japan). At least 5 random visual areas were chosen in every slide. The percentage and the integrated optical density (IOD) index of positively stained regions were scanned and analyzed using Image-Pro Plus (IPP) 6.0 software (Media Cybernetics Inc., Rockville, MD, USA). The penetration distance between the vessel and where the fluorescence decreased to background levels was also analyzed using the IPP software.

In vivo efficacy studies. When the tumors reached $\sim 100 \mathrm{~mm}^{3}$, the mice were divided into 4 groups ( $n=6 /$ group). The same injections as described for the tumor permeability assay were performed twice a week for a total of 8 times. The volume of the tumors was measured in two perpendicular directions with a caliper, and the weight of the mice was calculated every 3 days. The volume of the tumor was measured using the following formula: $\mathrm{V}=0.5 \mathrm{x}\left(\mathrm{W}^{2} \mathrm{xL}\right)$; where $\mathrm{V}=$ tumor volume, $\mathrm{W}=$ the smaller perpendicular diameter and $\mathrm{L}=$ the larger perpendicular diameter. On day 30 following the first injection, the mice were sacrificed and the tumors were collected and weighed. The tumor growth inhibition ratio (TGIR) was calculated using the formula: $T G I R=\left(W-W_{t}\right) / W \times 100$; where $\mathrm{W}$ is the average tumor weight of the PBS group and $\mathrm{W}_{t}$ is the average tumor weight of the treatment group. Each tumor was cut into two equal parts: one part was fixed in $10 \%$ neutral-buffered formalin for immunohistochemical staining and the TdT-mediated dUTP nick end labeling (TUNEL) assay; and the other part was snap-frozen in liquid nitrogen for western blot analysis.

Immunohistochemical staining. The fixed tumor tissues were embedded in paraffin and cut into 3-5 $\mu \mathrm{m}$ sections (20). The experiment was performed on with a streptavidin-peroxidase system (OriGene Technologies, Inc., Beijing, China). EGFR and phosphorylated EGFR (p-EGFR) were detected with a rabbit anti-human EGFR antibody (cat. no. ab52894; Abcam) and a rabbit anti-human p-EGFR antibody (cat. no. 36-9700; Thermo Fisher Scientific, Inc.), respectively. Imaging was performed by fluorescence microscopy (DS-Ri1; Nikon Corporation). To determine the IOD indices of EGFR and p-EGFR, 5 representative visual areas that were positive for EGFR or p-EGFR were examined from each tumor. The EGFR and p-EGFR indices for each selected area were analyzed using IPP 6.0 software.

Western blot analysis. Total protein was extracted using radioimmunoprecipitation assay buffer (Beyotime Institute of Biotechnology, Nanjing, China) containing $1 \mathrm{mM}$ phenylmethylsulfonyl fluoride (Sigma-Aldrich). Protein lysates were separated by $10 \%$ SDS-PAGE, followed by transfer to polyvinylidene difluoride membranes. The membranes were blocked with PBS containing 0.01\% Tween-20 (PBST) and 5\% $(\mathrm{w} / \mathrm{v})$ fat-free dried milk for $1 \mathrm{~h}$, followed by incubation with anti-EGFR and anti-p-EGFR antibodies (see above), and rabbit anti- $\beta$-actin polyclonal antibody (cat. no. ab8227; Abcam) for $1 \mathrm{~h}$. Subsequently, the membranes were washed five times with PBST and incubated with IRDye $800 \mathrm{CW}$ goat anti-rabbit IgG (H+L) antibody (cat. no. 926-32211; LI-COR Biosciences, Lincoln, NE, USA) for $1 \mathrm{~h}$. $\beta$-actin was detected as an internal control. After washing the membranes another five times with PBST, images were captured using an Odyssey enhanced chemiluminescence system (LI-COR Biosciences) and the intensity of the bands was quantified by ImageJ2x 2.1.4.7 software (Rawak Software, Inc., Stuttgart, Germany).

TUNEL assay. The paraffin sections were prepared, as described in the immunohistochemical staining section. The apoptotic cells were detected using a TUNEL assay kit (cat. no. 1684809; Roche Diagnostics), according to the manufacturer's protocol. Imaging was performed by fluorescence microscopy (DS-Ri1). The number of TUNEL-positive cells in 5 randomly selected fields from each tumor was counted. The percent of TUNEL-positive cells was calculated as the apoptotic index. A total of 100 cells were randomly selected for each field.

Statistical analysis. SPSS version 16.0 for Windows (SPSS, Inc., Chicago, IL, USA) was used for all analyses. Quantitative data were presented as the mean \pm standard deviation. Comparisons between two groups were performed using independent-sample Student's $t$-tests, while multiple samples were compared with one-way analysis of variance, with $\alpha=0.05$ as a level for the test. Results with a P-value of $<0.05$ were considered to indicate a statistically significant difference.

\section{Results}

Expression of $\alpha v \beta 3, \alpha v \beta 5, N R P-1$ and EGFR in A549 cells and xenograft tissue. The tumor-penetrating ability of iRGD mainly depends on the level of $\alpha v \beta 3, \alpha v \beta 5$ and NRP-1 in cancer cells $(18,21)$. The therapeutic efficacy of cetuximab mainly depends on the level of EGFR in cancer cells (22). 
A

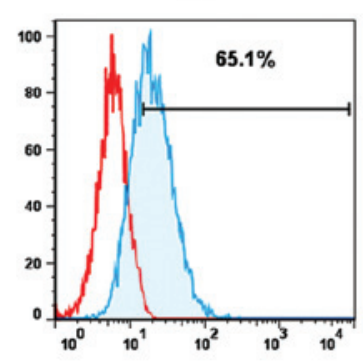

$\alpha v \beta 5$

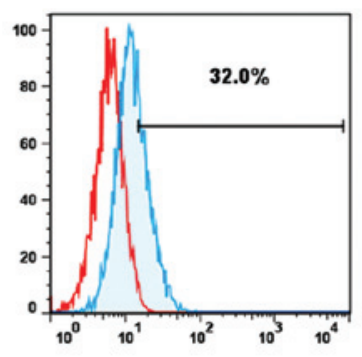

NRP-1

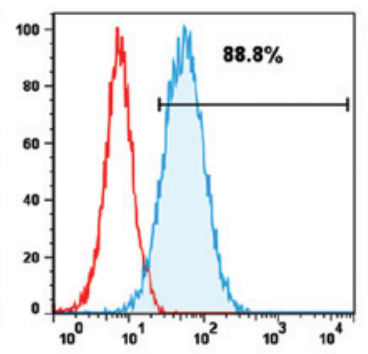

EGFR

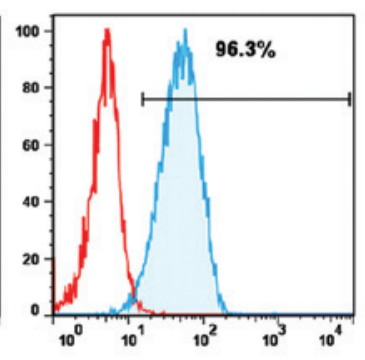

B

$\alpha v \beta 3$

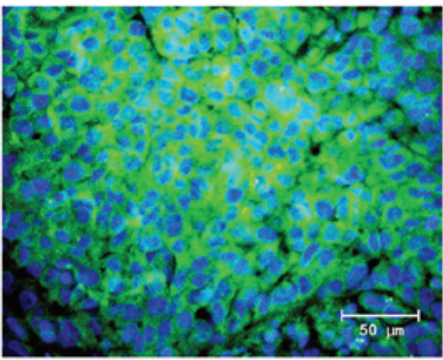

$\alpha v \beta 5$
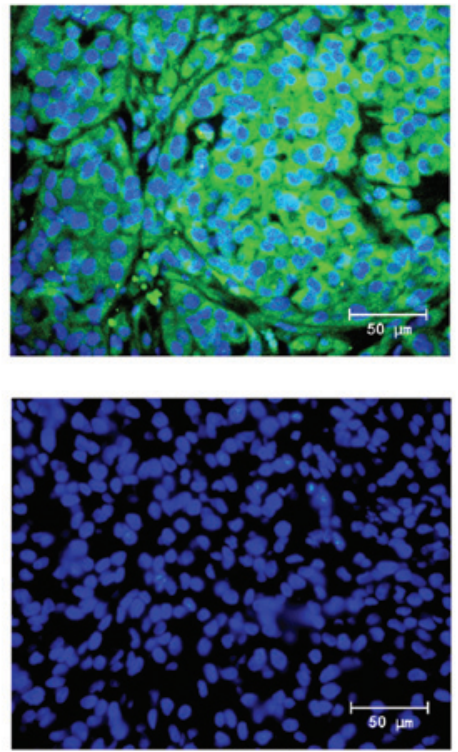

NRP-1

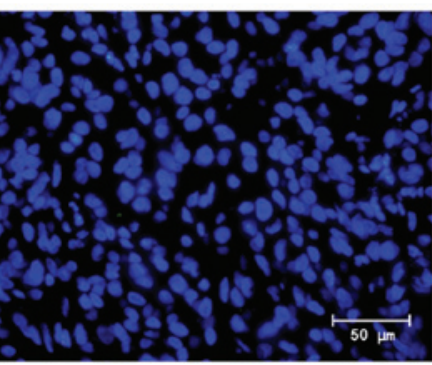

Figure 1. Expression of $\alpha v \beta 3, \alpha v \beta 5$, NRP-1 and EGFR in A549 cells and xenografts. (A) $\alpha v \beta 3, \alpha v \beta 5$, NRP-1 and EGFR expression in A549 cells was determined by flow cytometry. (B) $\alpha v \beta 3$, $\alpha v \beta 5$, NRP-1 and EGFR expression in mouse xenografts was determined by immunofluorescence staining. NRP-1, neuropilin-1; EGFR, epidermal growth factor receptor; EG, experimental group; CG, control group. Magnification, $\mathrm{x} 400$; Scale bars=50 $\mu \mathrm{m}$.

In order to confirm the expression of these molecules in the human NSCLC A549 cell line, a flow cytometry analysis was performed. As shown in Fig. 1A, the positive expression rates of $\alpha v \beta 3, \alpha v \beta 5$, NRP-1 and EGFR were 65.1, 32.0, 88.8 and $96.3 \%$, respectively. This result demonstrated that the A549 cell line may be used to establish a human NSCLC model for the study of the combination therapy of cetuximab and iRGD. To confirm the level of $\alpha v \beta 3$, $\alpha v \beta 5$, and NRP- 1 in tumor tissue, a BALB/c nude mouse xenograft model was developed with the A549 cell line. The expression of these molecules in tumor tissue was additionally detected by immunofluorescence staining. As shown in Fig. 1B, $\alpha v \beta 3$ (left), $\alpha v \beta 5$ (middle) and NRP-1 (right) were overexpressed in the xenograft tissues.

Tumor-penetrating ability of cetuximab combined with $i R G D$. To determine whether iRGD can promote the penetration of cetuximab into the human NSCLC xenograft, cetuximab that penetrated into the tumor tissue was detected by immunofluorescence staining. As shown in Fig. 2A, much more cetuximab could be observed in the cetuximab+iRGD group compared with in the cetuximab group, particularly after 3 and $9 \mathrm{~h}$ (Fig. 2A). The results of the quantitative analysis showed that the penetrating depths of cetuximab into the tumors in the cetuximab+iRGD group were 2.0,2.3 and 2.0 times the cetuximab only group; that the diffusing areas were 3.2, 3.5 and 2.4 times the cetuximab only group; and the accumulated amounts were 2.9,3.8 and 4.1 times the cetuximab only group at 1,3 and $9 \mathrm{~h}$ of treatment, respectively (Fig. 2B). These differences were statistically significant.

Therapeutic efficacy of cetuximab co-administered with $i R G D$. In order to determine an appropriate dose, at which cetuximab efficiently inhibits tumor growth without evident side effects, 3 doses of cetuximab (10,30 and $90 \mathrm{mg} / \mathrm{kg}$ ) were applied to treat 3 groups of mice $(n=3)$ via tail vain, twice per week for two consecutive weeks. After two weeks, the mice in that 10 and $30 \mathrm{mg} / \mathrm{kg}$ groups appeared normal, but the mice in the $90 \mathrm{mg} / \mathrm{kg}$ group showed an apparent lack of energy and loss of appetite. According to a previous study, the A549 xenograft in mice is sensitive to cetuximab at doses of $4 \mathrm{mg} / \mathrm{kg}$ and $40 \mathrm{mg} / \mathrm{kg}$ (22). Taking the efficacy and side effects into consideration, the present study chose $30 \mathrm{mg} / \mathrm{kg}$ as the dose of cetuximab in the in vivo therapeutic efficacy experiments.

To determine whether the therapeutic efficacy of cetuximab is enhanced when it is co-administered with iRGD, cetuximab and iRGD were co-administered to treat the mice with A549 xenografts. As shown in Fig. 3A, the tumors of the cetuximab+iRGD 
A

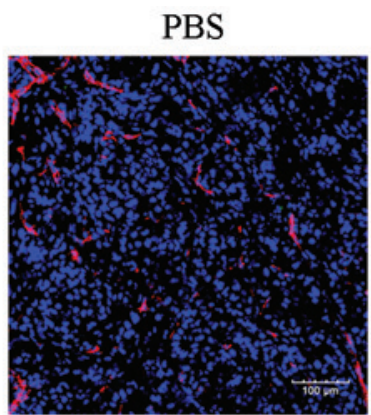

$1 \mathrm{~h}$

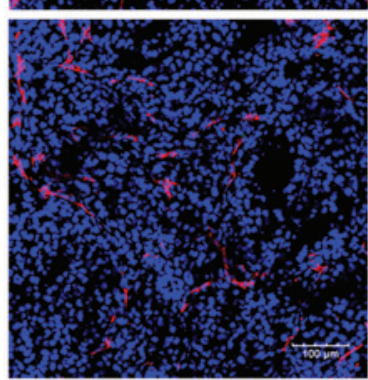

$3 \mathrm{~h}$

$9 \mathrm{~h}$

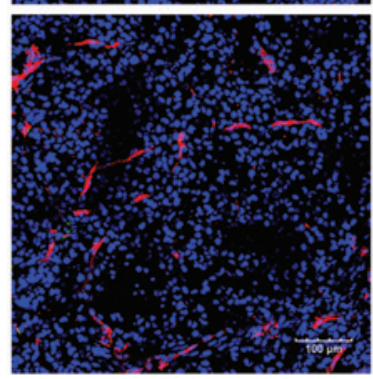

B

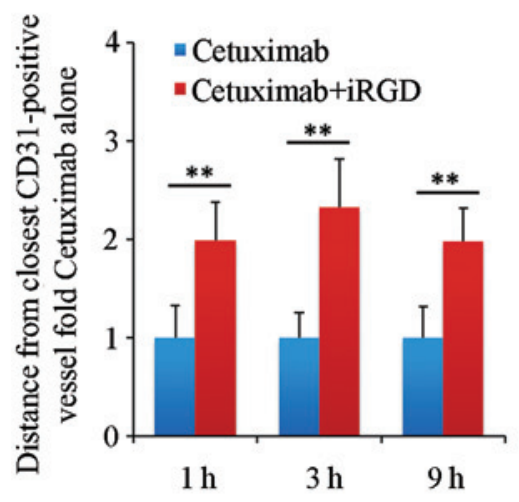

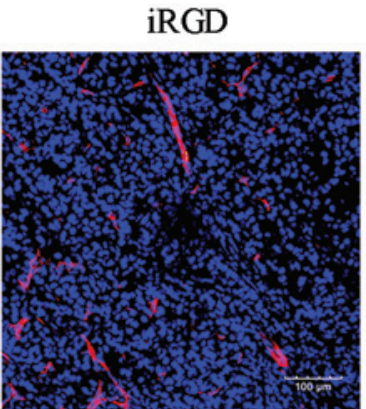
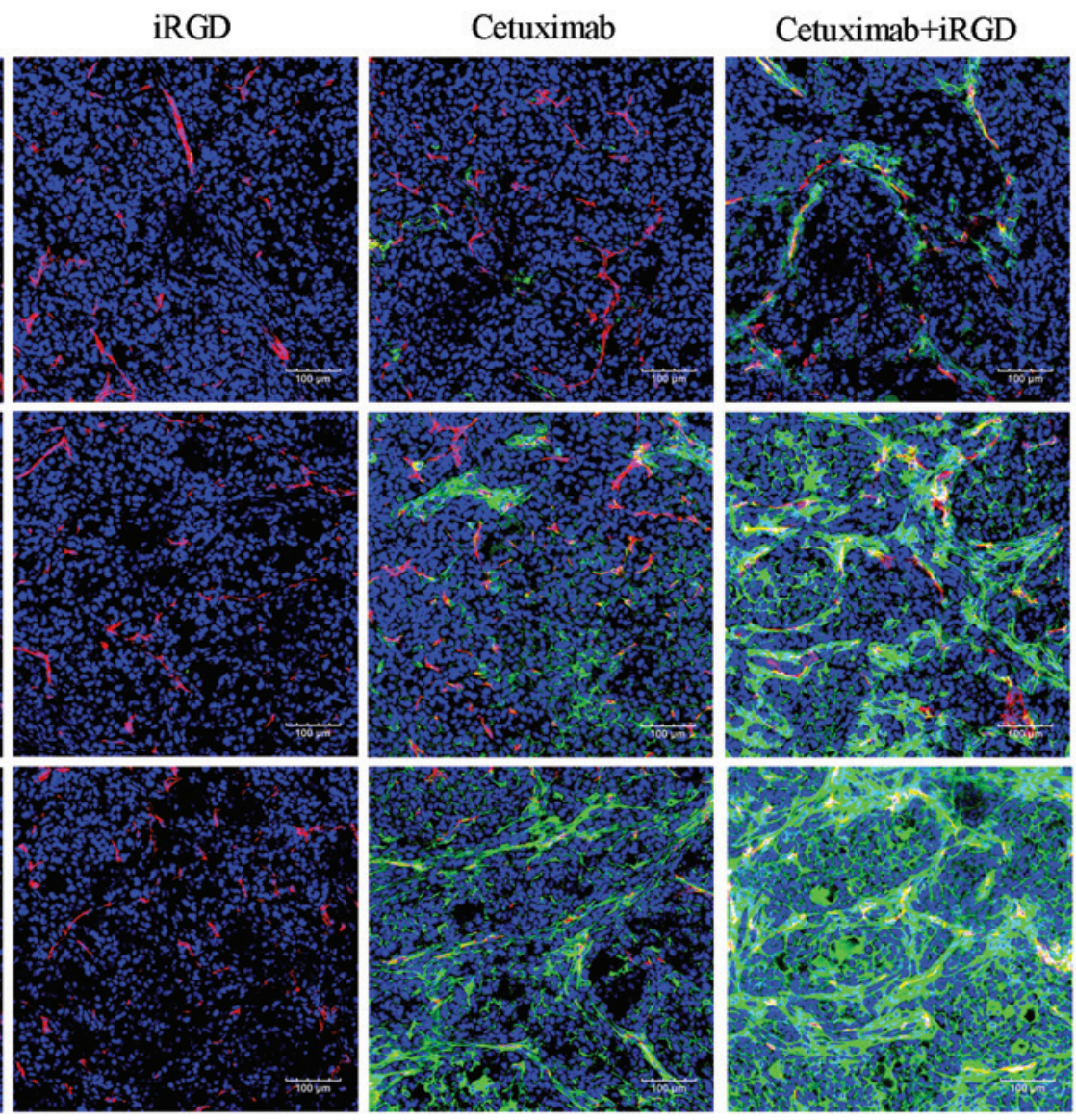

C

D

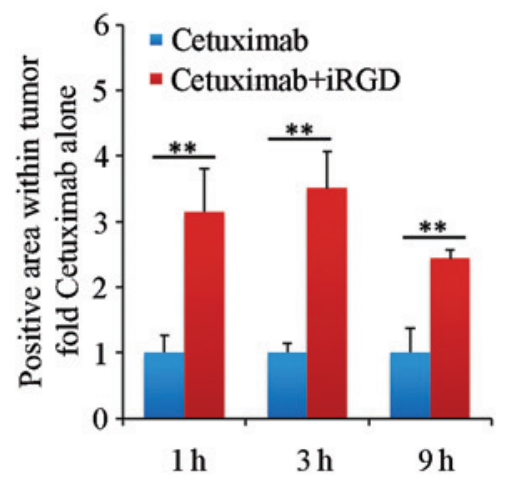

Figure 2. Penetration analysis of cetuximab in extravascular tumor tissues. (A) Immunofluorescence staining analysis. Tumor vasculature was stained with an anti-mouse cluster of differentiation 31 antibody. Cetuximab was stained with an anti-human IgG antibody. The nuclei were stained with 4',6-diamidino-2-phenylindole. Representative images from 3 tumors per group are shown; magnification, x200; Scale bars=100 $\mu \mathrm{m}$. (B) Quantification analysis of the penetrating depth of cetuximab in tumor tissues. (C) Quantitative analysis of the diffusing area of cetuximab in tumor tissues. (D) Quantitative analysis of the accumulated amount of cetuximab in tumor tissues. Five randomly selected fields in each section per tumor were analyzed with Image-Pro Plus software. $\mathrm{n}=3$; Error bars, mean \pm standard deviation; ${ }^{* *} \mathrm{P}<0.01 ;{ }^{* * *} \mathrm{P}<0.001$. PBS, phosphate-buffered saline; iRGD, internalizing RGD peptide.

group grew more slowly compared with the tumors in the cetuximab group, and this difference was statistically significant. The analysis of average tumor weight further confirmed this result (Fig. 3B). These results demonstrated that iRGD could enhance the therapeutic efficacy of cetuximab for A549 xenografts in mice. The body weight shift analysis of the experimental mice showed that no significant difference was observed between the cetuximab group and the cetuximab+iRGD group (Fig. 3C). These results demonstrated that iRGD did not evidently exacerbate the side effects of cetuximab.
EGFR phosphorylation in the tumors treated with cetuximab plus $i R G D$. With regards to the aforementioned results, iRGD promoted the penetration of cetuximab into tumor tissue and enhanced the therapeutic efficacy of cetuximab for the A549 xenograft. Therefore, theoretically, the co-administration of cetuximab and iRGD could result in the increased inhibition of EGFR phosphorylation compared with cetuximab monotherapy. In order to confirm this speculation, the present study first detected the phosphorylation level of EGFR in the tumor tissues by immunohistochemical staining. The expression 
A

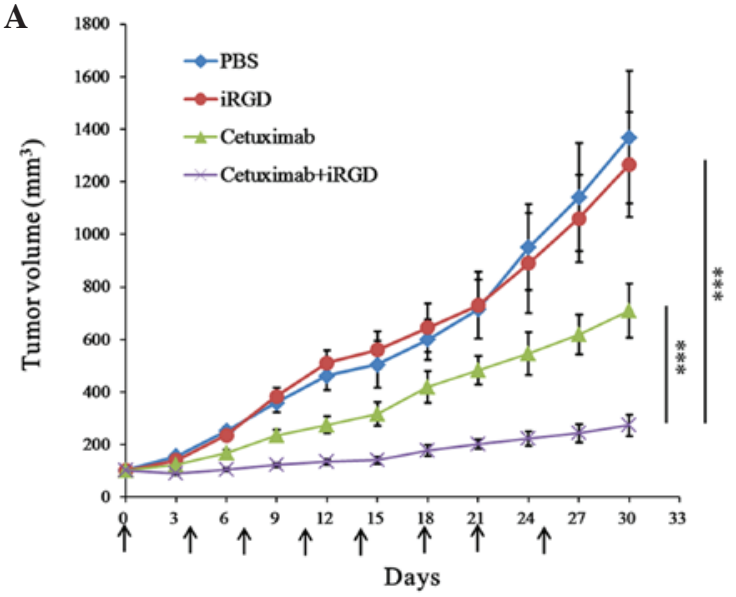

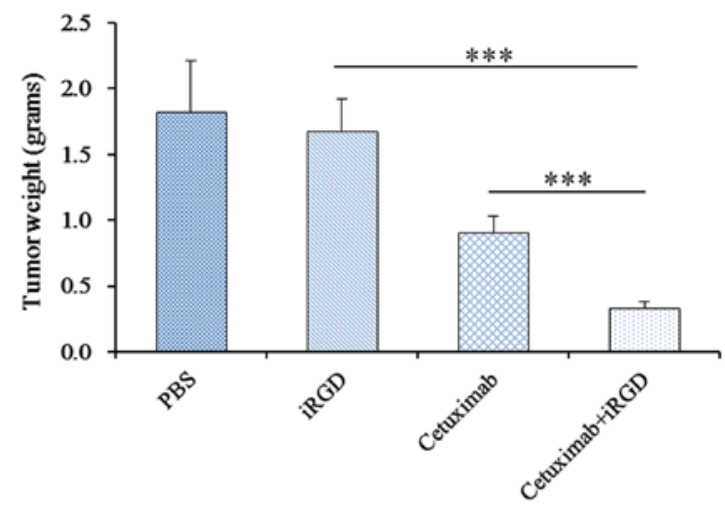

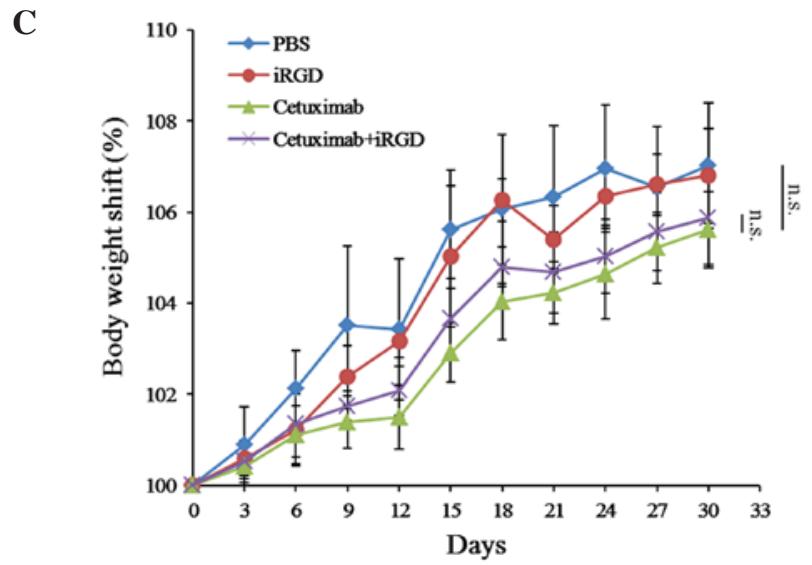

Figure 3. Therapeutic efficacy of Cetuximab co-administered with iRGD in A549 xenograft models. (A) Tumor growth curve during treatment. Arrows indicate the time of injection. The day when treatment started was recorded as day 0. Tumor volume was measured once every 3 days until day 30 . (B) Average tumor weight of each group at the end of the experiment. (C) Body weight shift curve of the mice during the experiment. $n=6$. Error bars, mean \pm standard deviation; ns, not significant; ${ }^{* * *} \mathrm{P}<0.001$. PBS, phosphate-buffered saline; iRGD, internalizing RGD peptide.

levels of EGFR among the 4 groups had no significant difference (Fig. 4A and B); however, the phosphorylation of EGFR in the cetuximab+iRGD group was reduced by $57.1 \%$ compared with the cetuximab group, which was statistically significant (Fig. 4A and C).

To further confirm the expression level of EGFR and p-EGFR, the total proteins were extracted from the tumor tissues in each group. The expression levels of EGFR and p-EGFR were detected by western blot analysis with $\beta$-actin as an internal control. Each strip was scanned and a quantitative analysis was performed with ImageJ software. The expression level of EGFR in the tumor tissues of the 4 groups had no significant difference (Fig. 4D and E); however, the expression level of p-EGFR in the cetuximab+iRGD group was reduced by $82.6 \%$ compared with the cetuximab group (Fig. 4D and F). Overall, these results confirmed that the therapeutic efficacy of cetuximab was enhanced when used in combination with the tumor-penetrating peptide iRGD.

Induction of apoptosis in the tumors treated with cetuximab plus $i R G D$. Previous studies have shown that cetuximab can induce cancer cell apoptosis by competitively blocking the binding of EGFR to its natural ligands $(9,23)$. Therefore, the present study detected the apoptosis of the cancer cells in the mice treated with A549 xenografts using a TUNEL assay. As shown in Fig. 5A, more apoptotic cells were visible in tumors of the cetuximab+iRGD group compared with tumors of the cetuximab group. The apoptotic index was defined as the percentage of TUNEL-positive cells vs. the total number of cells. According to the statistical analysis shown in Fig. 5B, the rate of apoptosis in tumors of the cetuximab+iRGD group was 2.1 times the apoptosis rate in tumors of the cetuximab group. These results further confirmed that iRGD enhanced the therapeutic efficacy of cetuximab in the human NSCLC xenograft.

\section{Discussion}

The present study was conducted to evaluate the use of iRGD for co-administration therapy with cetuximab, and demonstrated that iRGD boosted the accumulation of cetuximab in human NSCLC xenograft models established with the A549 cell line. The anticancer effects of cetuximab were also enhanced by iRGD co-administration in this NSCLC model. To further verify the effects of drug accumulation and anticancer, the phosphorylation of EGFR and cell apoptosis were detected in the treated tumors.

The experiments using A549 xenograft models confirmed the enhanced cetuximab accumulation effect and therapeutic efficacy of iRGD. Previous reports demonstrated that the drug accumulation effect of iRGD is dependent on the level 
A

PBS

iRGD

Cetuximab

Cetuximab+iRGD

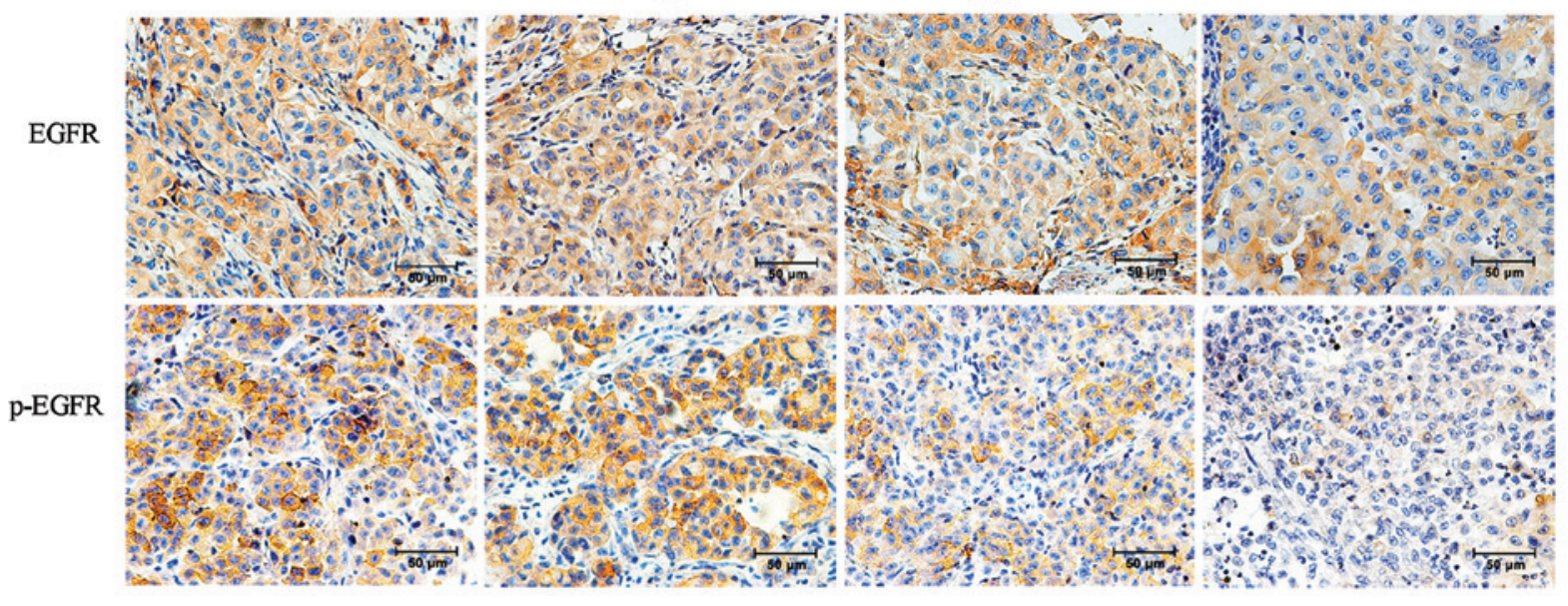

B

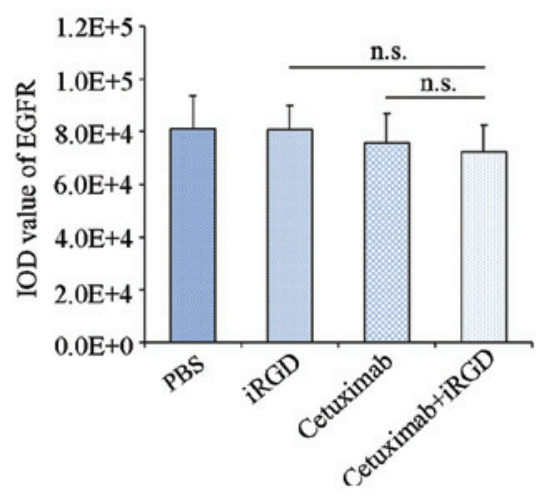

C

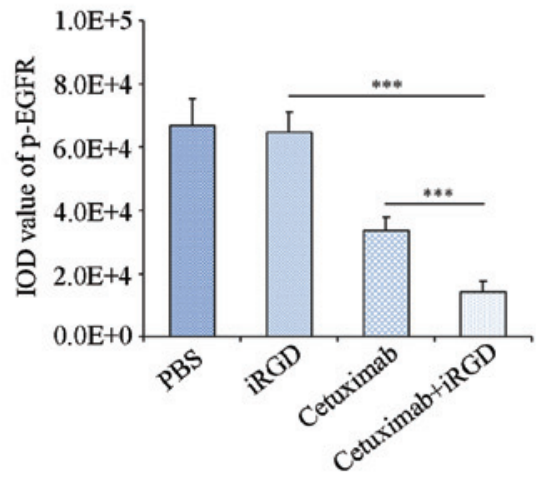

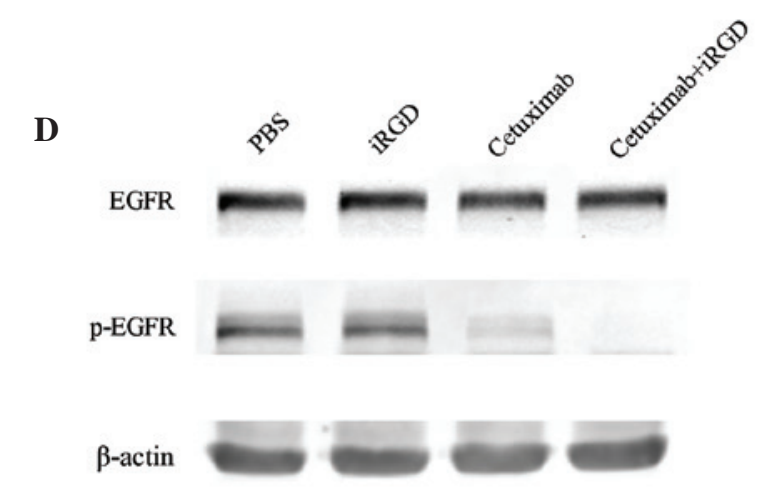

$\mathbf{E}$

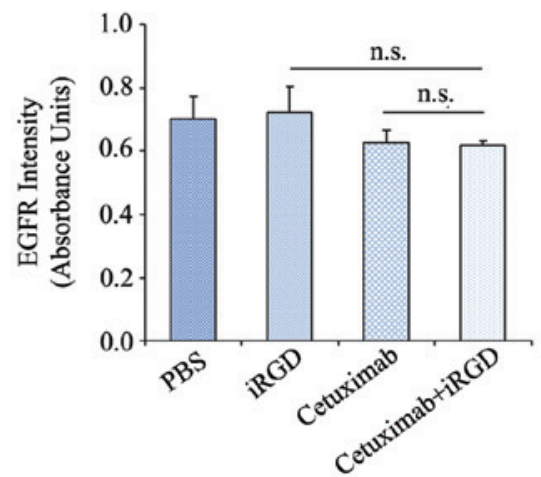

F

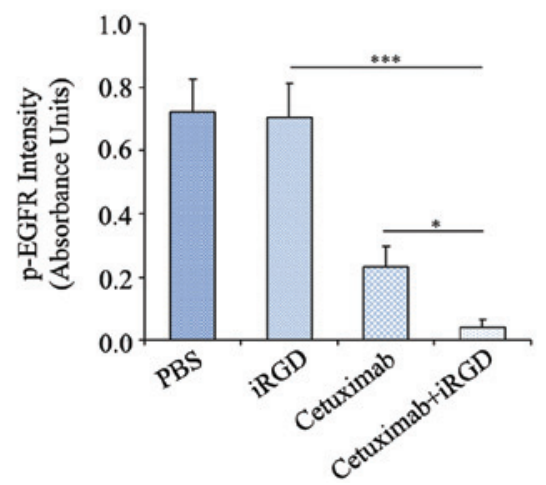

Figure 4. The inhibition of EGFR phosphorylation following co-administration of cetuximab and iRGD. (A) Immunohistochemical staining analysis. EGFR and p-EGFR positive cells in the sections are stained brown. The nuclei are stained blue. Representative figures from each group are shown; $\mathrm{n}=6$; magnification, $\mathrm{x} 400$; Scale bars $=50 \mu \mathrm{m}$. Quantitative analysis results of (B) EGFR and (C) p-EGFR for part (A). Five fields of each tumor tissue section were randomly selected for the calculation of the IOD value of the positive region through Image-Pro Plus software, which indicated the amount of antigen expression. (D) Western blot analysis. Total protein from the tumor tissues was extracted. EGFR and p-EGFR were detected with $\beta$-actin as an internal control. $n=3$. Quantitative analysis results of (E) EGFR and (F) p-EGFR of part (D). The intensity of each strip was analyzed by ImageJ software. The average intensities of EGFR and p-EGFR were standardized to $\beta$-actin. Error bars, mean \pm standard deviation; ns, not significant; ${ }^{*} \mathrm{P}<0.05 ;{ }^{* * *} \mathrm{P}<0.001$. EGFR, epidermal growth factor receptor; $\mathrm{p}$-EGFR, phosphorylated-epidermal growth factor receptor; PBS, phosphate-buffered saline; iRGD, internalizing RGD peptide; IOD, integrated optical density. 
A
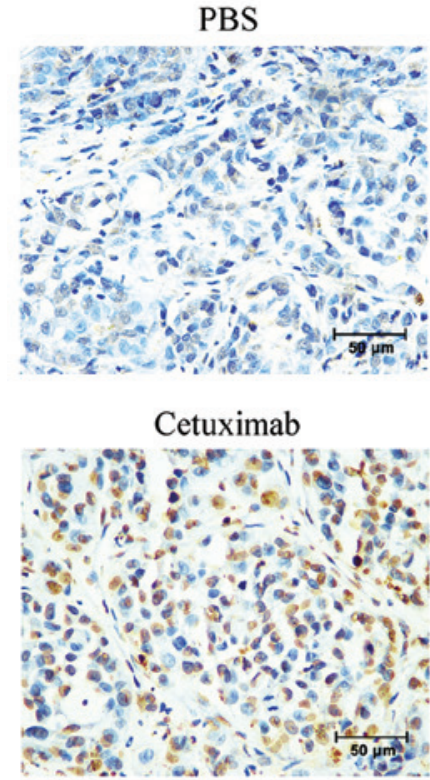

iRGD

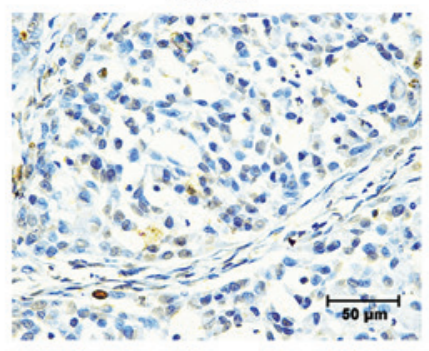

Cetuximab+iRGD

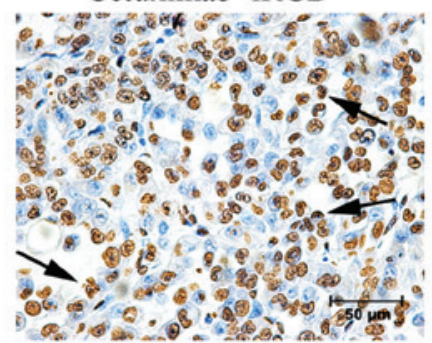

B

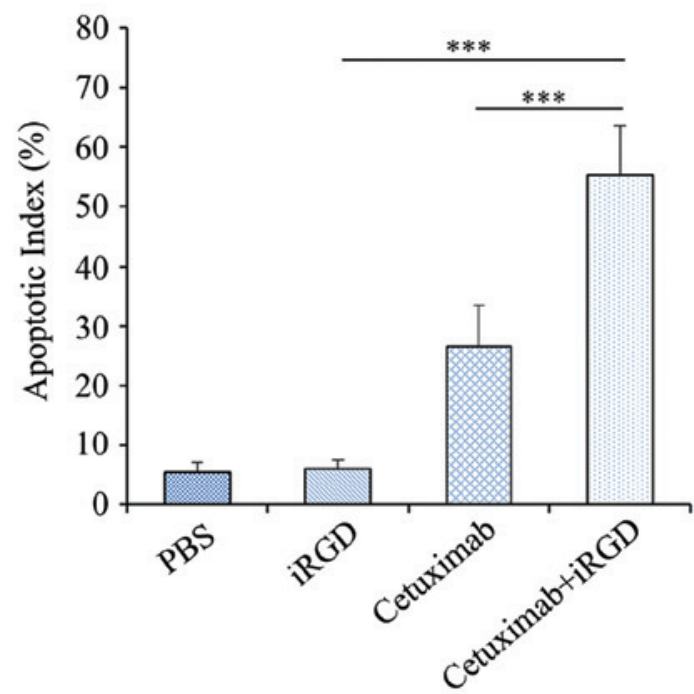

Figure 5. Cancer cell apoptotic in the tumor co-administered with cetuximab and iRGD. (A) Apoptotic cells in the tumor tissues were detected by a TUNEL assay. TUNEL-positive nuclei are stained brown. TUNEL-negative nuclei are stained blue. The figures shown here are representative of the six tumors in each group. Arrows indicate the apoptotic bodies. Magnification, $x 400$; Scale bars $=50 \mu \mathrm{m}$. (B) Quantitative analysis of the apoptosis index in each group. The percentage of TUNEL-positive cells counted from 100 randomly selected tumor cells per section. Five sections were counted per tumor. $\mathrm{n}=6$; Error bars, mean \pm standard deviation; ${ }^{* * *} \mathrm{P}<0.001$. PBS, phosphate-buffered saline; iRGD, internalizing RGD peptide; TUNEL, TdT-mediated dUTP nick end labeling kit.

of $\alpha v \beta 3, \alpha v \beta 5$ and NRP-1 in the tumor tissue $(14,18,24)$. The results of the present study showed that these molecules are overexpressed in A549 cells and in the xenograft tissue. Furthermore, cetuximab accumulation was detected in tumor tissues. The results showed that the accumulation of cetuximab in tumor tissue is enhanced by iRGD. Theoretically, the enhanced accumulation of cetuximab in tumor tissues could result in decreased EGFR phosphorylation, increased cell apoptosis and inhibited tumor growth. The results of the current study were consistent with these theoretical speculations. Therefore, the results confirmed that iRGD can enhance the tumor-penetration and therapeutic efficacy of cetuximab in A549 xenograft models.

It is possible that combination with iRGD enhances the therapeutic efficacy and decreases the side effects of cetuximab. The results of a single-agent, phase II study in recurrent NSCLC ( $n=29)$ showed limited therapeutic efficacy; $7 \%$ of patients showed a partial response and $17 \%$ patients showed stable disease (8). In one phase III trial, cetuximab was evaluated in combination with docetaxel and pemetrexed in patients with advanced NSCLC, and the results indicated that cetuximab does not add any benefit to second line chemotherapy for patients with NSCLC $(25,26)$. The BMS099 trial also failed to show a significant improvement in progression-free survival (5). In the present study, iRGD was combined with cetuximab to treat an NSCLC xenograft model, and the results indicated that iRGD can enhance the therapeutic efficacy of cetuximab for NSCLC without evident side effects at dose of $30 \mathrm{mg} / \mathrm{kg}$. Therefore, it is possible that iRGD may add benefit to cetuximab for patients with NSCLC. However, the efficacy of iRGD has only been examined in the A549 cell line, so the clinical applications of these findings require careful consideration.
The use of cetuximab and iRGD combination therapy may have certain problems if it is applied clinically. The current understanding of the mechanism by which iRGD enhances the therapeutic efficacy of cetuximab for NSCLC is limited. Although no evident side effects were observed in the present study, the safety of this combination therapy requires additional evaluation. The CendR motif of iRGD may also be used by viruses and microbial toxins in order to gain entry into cells and spread within the tissues of the body (27-29). Since EGFR is also overexpressed in novel vessels, iRGD also may promote the uptake of cetuximab into normal tissues that are damaged, and the tissue repair that follows may interfere with the normal function of EGFR and cause further damage to the body.

In summary, through a human NSCLC A549 nude mouse xenograft model, iRGD was confirmed to promote the penetration of cetuximab into tumor tissue and therefore enhance the therapeutic efficacy of cetuximab in vivo. The combined application of cetuximab and iRGD may be a novel strategy to enhance the clinical therapeutic efficacy of cetuximab for the treatment of NSCLC.

\section{Acknowledgements}

The present study was supported by the National Natural Science Foundation of China (Beijing, China; grant no. 81301946), Natural Science Foundation of Jiangsu Province (Nanjing, China; grant no. BK2012146), Jiangsu Provincial Office of Education Foundation (Nanjing, China; grant no. JHB2012-34), Xuzhou Medical College Foundation (Xuzhou, China; grant no. 2012KJZ23) and 'Liu Da Ren Cai Gao Feng' Foundation of Jiangsu Province (Nanjing, China; grant no. 53031305). 


\section{References}

1. Privitera G, Luca T, Musso N, Vancheri C, Crimi N, Barresi V, Condorelli D and Castorina S: In vitro antiproliferative effect of trastuzumab (Herceptin ${ }^{\circledR}$ ) combined with cetuximab (Erbitux $\left.{ }^{\circledR}\right)$ in a model of human non-small cell lung cancer expressing EGFR and HER2. Clin Exp Med, 2015.

2. Patil N, Abba M and Allgayer H: Cetuximab and biomarkers in non-small-cell lung carcinoma. Biologics 6: 221-231, 2012.

3. Pujol JL, Pirker R, Lynch TJ, Butts CA, Rosell R, Shepherd FA, Vansteenkiste J, O'Byrne KJ, de Blas B, Heighway J, et al: Meta-analysis of individual patient data from randomized trials of chemotherapy plus cetuximab as first-line treatment for advanced non-small cell lung cancer. Lung Cancer 83: 211-218, 2014.

4. Kim SM, Kim JS, Kim JH, Yun CO, Kim EM, Kim HK, Solca F, Choi SY and Cho BC: Acquired resistance to cetuximab is mediated by increased PTEN instability and leads cross-resistance to gefitinib in HCC827 NSCLC cells. Cancer Lett 296: 150-159, 2010.

5. Pirker R, Pereira JR, von Pawel J, Krzakowski M, Ramlau R, Park K, de Marinis F, Eberhardt WE, Paz-Ares L, Störkel S, et al: EGFR expression as a predictor of survival for first-line chemotherapy plus cetuximab in patients with advanced non-small-cell lung cancer: Analysis of data from the phase 3 FLEX study. Lancet Oncol 13: 33-42, 2012.

6. Douillard JY, Pirker R, O'Byrne KJ, Kerr KM, Störkel S, von Heydebreck A, Grote HJ, Celik I and Shepherd FA: Relationship between EGFR expression, EGFR mutation status and the efficacy of chemotherapy plus cetuximab in FLEX study patients with advanced non-small-cell lung cancer. J Thorac Oncol 9: 717-724, 2014.

7. van den Heuvel MM, Uyterlinde W, Vincent AD, de Jong J, Aerts J, Koppe F, Knegjens J, Codrington H, Kunst PW, Dieleman E, et al: Additional weekly Cetuximab to concurrent chemoradiotherapy in locally advanced non-small cell lung carcinoma: Efficacy and safety outcomes of a randomized, multi-center phase II study investigating. Radiother Oncol 110: 126-131, 2014.

8. Dubey S and Schiller JH: Three emerging new drugs for NSCLC: Pemetrexed, bortezomib and cetuximab. Oncologist 10: 282-291, 2005.

9. Wild R, Fager K, Flefleh C, Kan D, Inigo I, Castaneda S, Luo FR, Camuso A, McGlinchey K and Rose WC: Cetuximab preclinical antitumor activity (monotherapy and combination based) is not predicted by relative total or activated epidermal growth factor receptor tumor expression levels. Mol Cancer Ther 5: 104-113, 2006

10. Yu T, Wang Z, Liu K, Wu Y, Fan J, Chen J, Li C, Zhu G and Li L: High interstitial fluid pressure promotes tumor progression through inducing lymphatic metastasis-related protein expressions in oral squamous cell carcinoma. Clin Transl Oncol 16: 539-547, 2014.

11. Milosevic MF, Pintilie M, Hedley DW, Bristow RG, Wouters BG, Oza AM, Laframboise S, Hill RP and Fyles AW: High tumor interstitial fluid pressure identifies cervical cancer patients with improved survival from radiotherapy plus cisplatin versus radiotherapy alone. Int J Cancer 135: 1692-1699, 2014.

12. Trédan O, Galmarini CM, Patel K and Tannock IF: Drug resistance and the solid tumor microenvironment. J Natl Cancer Inst 99: 1441-1454, 2007.

13. Song W, Li M, Tang Z, Li Q, Yang Y, Liu H, Duan T, Hong H and Chen X: Methoxypoly (ethylene glycol)-block-poly (L-glutamic acid)-loaded cisplatin and a combination with iRGD for the treatment of non-small-cell lung cancers. Macromol Biosci 12: $1514-1523,2012$
14. Sugahara KN, Teesalu T, Karmali PP, Kotamraju VR, Agemy L, Girard OM, Hanahan D, Mattrey RF and Ruoslahti E: Tissue-penetrating delivery of compounds and nanoparticles into tumors. Cancer Cell 16: 510-520, 2009.

15. Su S, Wang H, Liu X, Wu Y and Nie G: iRGD-coupled responsive fluorescent nanogel for targeted drug delivery. Biomaterials 34 : 3523-3533, 2013.

16. Alberici L, Roth L, Sugahara KN, Agemy L, Kotamraju VR, Teesalu T, Bordignon C, Traversari C, Rizzardi GP and Ruoslahti E: De novo design of a tumor-penetrating peptide. Cancer Res 73: 804-812, 2013.

17. Roth L, Agemy L, Kotamraju VR, Braun G, Teesalu T, Sugahara KN, Hamzah J and Ruoslahti E: Transtumoral targeting enabled by a novel neuropilin-binding peptide. Oncogene 31 : 3754-3763, 2012.

18. Sugahara KN, Teesalu T, Karmali PP, Kotamraju VR, Agemy L, Greenwald DR and Ruoslahti E: Coadministration of a tumor-penetrating peptide enhances the efficacy of cancer drugs. Science 328: 1031-1035, 2010.

19. Zhang Q, Liu X, Xu S, Li C, Zhang Y, Yang J and Zheng J: Factor VII light chain-targeted lidamycin shows intensified therapeutic efficacy for liver cancer. Cancer Biother Radiopharm 27: 384-391, 2012.

20. Aizawa K, Liu C, Veeramachaneni S, Hu KQ, Smith DE and Wang XD: Development of ferret as a human lung cancer model by injecting 4-(Nmethyl-N-nitrosamino)-1-(3-pyridyl)-1-bu tanone (NNK). Lung Cancer 82: 390-396, 2013.

21. Ning L, Li X, Ding X, Yin Y and Li G: An iRGD based strategy to study electrochemically the species inside a cell. Int J Mol Sci 13: 10424-10431, 2012.

22. Steiner P, Joynes C, Bassi R, Wang S, Tonra JR, Hadari YR and Hicklin DJ: Tumor growth inhibition with cetuximab and chemotherapy in non-small cell lung cancer xenografts expressing wild-type and mutated epidermal growth factor receptor. Clin Cancer Res 13: 1540-1551, 2007.

23. Xu F, Tian Y, Huang Y, Zhang LL, Guo ZZ, Huang JJ and Lin TY: EGFR inhibitors sensitize non-small cell lung cancer cells to TRAIL-induced apoptosis. Chin J Cancer 30: 701-711, 2011.

24. Akashi Y, Oda T, Ohara Y, Miyamoto R, Kurokawa T, Hashimoto S, Enomoto T, Yamada K, Satake M and Ohkohchi N: Anticancer effects of gemcitabine are enhanced by co-administered $\mathrm{R}$ RDD peptide in murine pancreatic cancer models that overexpressed neuropilin-1. Br J Cancer 110: 1481-1487, 2014.

25. Villanueva MT: Lung cancer: Cetuximab, you're fired. Nat Rev Clin Oncol 11: 3, 2014.

26. Rasul KI and Kerr DJ: Targeted therapies: Cetuximab plus chemotherapy in patients with advanced NSCLC. Nat Rev Clin Oncol 6: 499-500, 2009

27. Sanchez AJ, Vincent MJ, Erickson BR and Nichol ST: Crimean-congo hemorrhagic fever virus glycoprotein precursor is cleaved by Furin-like and SKI-1 proteases to generate a novel 38-kilodalton glycoprotein. J Virol 80: 514-525, 2006.

28. Wool-Lewis RJ and Bates P: Endoproteolytic processing of the ebola virus envelope glycoprotein: Cleavage is not required for function. J Virol 73: 1419-1426, 1999.

29. Sjöberg M, Wallin M, Lindqvist B and Garoff H: Furin cleavage potentiates the membrane fusion-controlling intersubunit disulfide bond isomerization activity of leukemia virus Env. J Virol 80: 5540-5551, 2006. 